\title{
Atividades de extensão universitária em comitê de prevenção de mortalidade infantil e estatísticas de saúde
}

\author{
Activities of university extension in an infant mortality prevention committee and health statistics \\ Actividades de extensión universitaria en comité de prevención de la mortalidad infantil y estadísticas en salud
}

\section{Thais Aidar de Freitas Mathias', Taqueco Teruya Uchimura', Amanda Nolasco de Assunção', Kelen Marja Predebon'}

'Universidade Estadual de Maringá. Departamento de Enfermagem. Maringá, PR

Submissão: 10/04/2008

Aprovação: 20/02/2009

\section{RESUMO}

São descritas atividades de extensão no Comitê de Prevenção da Mortalidade Infantil da $15^{\text {a }}$ Regional de Saúde do Paraná e a aproximação com estatísticas de saúde. O projeto articula parceria entre o Departamento de Enfermagem da Universidade Estadual de Maringá e Secretaria de Saúde do Estado com inserção de alunos de enfermagem. São apresentadas as atribuições do Comitê, avanços desde o inicio do projeto, como a compreensão dos sistemas de informação e importância da investigação do óbito infantil, mas existem desafios, como melhorar a Qualidade do preenchimento da ficha de investigação. A parceria ensino/serviço aprimora a Qualidade do trabalho, dos Sistemas de Informação, amplia a visão do aluno sobre desigualdades intraurbanas e acesso, incentivando seu comprometimento com a saúde coletiva.

Descritores: Mortalidade infantil; Sistemas de Informação; Comitê de profissionais; Serviços de integração docente-assistencial; Enfermagem em saúde pública.

\begin{abstract}
Extra classes' activities with the Committee of Infant Mortality Prevention of the 15th Paraná Health Office (15th HO) and some approaches to health statistics, are described. Those activities articulate a partnership between the Nursing Department of the State University of Maringá and the 15th HO. A description of the Committee's attributions, the advances since the partnership start, the importance of the infant mortality investigations as well as understanding the information systems are presented. There are still challenges such as to improve the Quality of the ineuiry form. The partnership University - 15th HO improves the Quality of the Information Systems, provides the students a wider perspective of the intra-urban inequalities to access to health services, stimulating their commitment with public health.
\end{abstract}

Descriptors: Infant mortality; Information systems; Professional staff committees; Teaching care integration services; Public health nursing.

\section{RESUMEN}

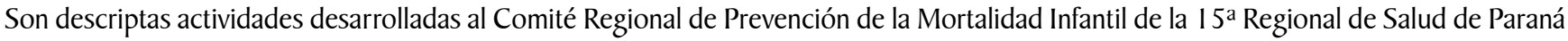

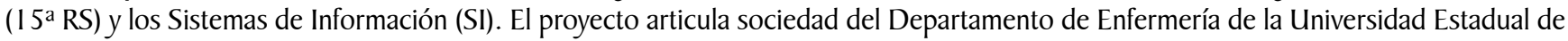
Maringá y la $15^{\text {a }}$ RS. Son presentadas atribuciones del Comité, dificultades en la investigación del óbito infantil, manoseo y comprensión, por los participantes, de los SI. Son presentados avances, pero existen desafíos como la calidad de la ficha de investigación del óbito. La sociedad enseñanza/servicio perfecciona la calidad del trabajo del Comité, de los SI y amplia la visión del alumno sobre desigualdades intra urbanas y acceso a servicios incentivando su formación más comprometida con la salud colectiva.

Descriptores: Mortalidad infantil, Sistemas de información; Comité de profesionales; Servicios de Integración Docente Asistencial; Enfermería en Salud Pública 


\section{INTRODUÇÃO}

Este relato tem por objetivo descrever atividades desenvolvidas em Projeto de Extensão Universitária, em especial destacar o trabalho conjunto dos participantes no Comitê Regional de Prevenção da

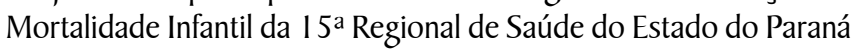
(CRPMI).

O projeto de extensão iniciou, oficialmente em junho de 2004 visando articular parceria entre a Secretaria Estadual de Saúde do Paraná (SESA/PR), Secretaria Municipal de Saúde de Maringá (SMS) e pelo Departamento de Enfermagem da Universidade Estadual de Maringá. Na SMS do município o trabalho é desenvolvido principalmente no Setor de Epidemiologia e na SESA/PR, no Setor de Epidemiologia da $15^{\text {a }}$ Regional de Saúde ( $\left.15^{\mathrm{a}} \mathrm{RS}\right)$. A equipe do projeto é composta por docentes do Departamento de Enfermagem, alunos de graduação e pós-graduação em Enfermagem além dos trabalhadores da SMS e $15^{\mathrm{a}} \mathrm{RS}$.

Tanto as atividades realizadas na SMS de Maringá com o Programa Bebê de Risco, a avaliação dos dados do SISPRENATAL nas Unidades Básicas de Saúde, como as realizadas na $15^{\text {a }}$ RS no Comitê, a aproximação dos participantes aos Sistemas de Informação em Saúde do Sistema Único de Saúde (SUS) e articulação ensinoserviço são fortemente estimuladas. A integração ensino-serviço contribui para o aprimoramento das atividades desenvolvidas nos serviços de saúde propiciando melhorar Qualitativa e Quantitativamente as informações coletadas e conseQüentemente os bancos de dados do SUS.

Com a inserção de alunos bolsistas de extensão, de iniciação científica e alunos de pós-graduação pretende-se também contribuir para a formação do enfermeiro já Que este profissional vem assumindo cada vez mais funções gerenciais em todos os níveis de atenção à saúde e, o planejamento, controle e avaliação tornaramse instrumentos necessários para o direcionamento das suas ações. Essas atribuições colocam o enfermeiro em contato com muitas situações complexas para as Quais deve apresentar soluções Que por sua vez, devem estar fundamentadas em informações, ingrediente fundamental do processo de decisão ${ }^{(1)}$.

Entre as atividades desenvolvidas no projeto de extensão destacam-se: avaliar e aprimorar o Programa Bebê de Risco no Setor de Epidemiologia da SMS de Maringá que tem como principal fonte o banco de dados do Sistema de Informação sobre Nascidos Vivos (Sinasc); avaliar e aprimorar os dados do Sistema de Informação do Pré-natal (SISPRENATAL), relativos aos relatórios resultantes do atendimento à gestante, realizado nas Unidades Básicas de Saúde do município de Maringá e atividades no Setor de Epidemiologia da $15^{\text {a }} \mathrm{RS}$ relativas às funções do Comitê Regional de Prevenção da Mortalidade Infantil (CRPMI). Na 15 $5^{\mathrm{a}} \mathrm{RS}$ as atividades envolvem processos de organização do trabalho do Comitê como a estruturação de planilhas, de apoio à coleta, recepção, análise e conclusão dos casos, digitação de dados e confecção de relatórios, discussão e participação nos estudos de caso do Comitê e sobre evitabilidade do óbito infantil. Além disso, o projeto articula as linhas de pesquisas dos programas de pósgraduação do DEN resultando na condução de projetos de pesQuisa e divulgação dos principais resultados em eventos e periódicos. $\mathrm{Na}$ $15^{\text {a }} \mathrm{RS}$ os bancos de dados utilizados são: o Sistema de Informação sobre Mortalidade (SIM), o Sistema de Informação sobre Nascidos
Vivos (Sinasc) e o Sistema de Informação de Mortalidade Infantil (SIMI).

\section{Consideraçoes sobre os Sistemas de Informação em Saúde para o SUS}

Nos últimos anos um dos principais desafios técnicos impostos ao SUS foi o desenvolvimento de mecanismos para seu aperfeiçoamento gerencial e ampliação do campo de atuação dos setores de vigilância à saúde, para promover atenção integral à saúde de fato para todos os segmentos da população, em especial aos mais carentes. Para esta finalidade tem-se intensificado a descentralização da maior parte das atividades aos estados e municípios incorporando às suas atribuições o uso da epidemiologia no seu cotidiano. Assim, as informações epidemiológicas têm sido cada vez mais utilizadas em nível regional e local para análise dos problemas de saúde e de suas causas ${ }^{(2)}$. (Epidemiologia nas Políticas, Programas e Serviços de Saúde, 2005). Para isso são necessários sistemas de informação Que reúnam os dados de eventos e agravos ocorridos na população Que sejam em Quantidade e Qualidade suficientes para Que as ações e planejamento em saúde sejam baseados em informações o mais próximo da realidade possível ${ }^{(3)}$.

Os Sistemas de Informação em Saúde (SIS) têm como função o planejamento, a coordenação e a supervisão dos processos de seleção, coleta, aQuisição, registro, armazenamento, processamento, recuperação, análise e difusão de dados e geração de informações. Os SIS constituem-se assim em componente do sistema de saúde para facilitar a formulação e avaliação das políticas, planos e programas de saúde, subsidiando o processo de tomada de decisões com vistas a contribuir para a melhoria da situação de saúde da população(4).

Os SIS com potencial para uso epidemiológico existentes na área da saúde em nível nacional Que se destacam em razão de sua maior relevância para a Vigilância Epidemiológica e Que podem ser utilizados em nível descentralizado são os de mortalidade (Sistema de Informações de Mortalidade-SIM), nascidos vivos (Sistema de Informações sobre Nascidos Vivos - SINASC) e agravos notificáveis (Sistema de Informações de Agravos sob Notificação - SINAN), além das internações hospitalares (Sistema de Informações Hospitalares - SIH-SUS) e consultas ambulatoriais (Sistema de Informações Ambulatoriais - SIS-SUS) ${ }^{(5)}$. Outros sistemas de informação complementares são desenvolvidos pelo Centro Nacional de Epidemiologia (CENEPI) tais como: Sistema de Informações de Vigilância Alimentar e Nutricional (SISVAN) Sistema de informações do programa nacional de imunização (SI-PNI).

Um dos objetivos básicos dos SIS na concepção do Sistema Único de Saúde (SUS) é possibilitar a análise da situação de saúde no nível local, tomando como referencial microrregiões homogêneas e considerando, necessariamente, as condições de vida da população na determinação do processo saúde-doença. O nível local tem então a responsabilidade não apenas com a alimentação dos SIS, mas também com a sua organização e gestão ${ }^{(6)}$. A produção das informações em saúde de forma ágil, atualizada, completa e fidedigna, vem se conformando num importante instrumento de controle social do SUS, na medida em Que possibilita à população o acompanhamento e a avaliação das atividades dos serviços de saúde ${ }^{(7)}$.

\section{O CRPMI e as Estatísticas de Saúde}

Em relação às informações geradas pelo setor saúde as de 
mortalidade são as mais tradicionais e Quando analisadas propiciam o conhecimento do perfil de saúde de uma determinada comunidade. Dos indicadores de mortalidade o de mortalidade infantil é classicamente reconhecido por refletir condições socioeconômicas da população além de ser considerado evento indicador da facilidade de acesso e da Qualidade dos serviços de saúde( ${ }^{(8)}$.

Quando ocorre um óbito infantil os Comitês da Prevenção de Mortalidade Infantil (CPMI) desenvolvem estudos de caso para investigar tal ocorrência. O Pacto Nacional pela Redução da Mortalidade Materna e Neonatal lançado em março de 2004 é prioridade do Ministério da Saúde e é compromisso do governo federal. Entre as iniciativas governamentais para a redução da mortalidade infantil evitável, constantes no Pacto, está a estruturação e aprimoramento das atividades desenvolvidas pelos Comitês de Prevenção da Mortalidade Infantil( ${ }^{(9)}$. De caráter eminentemente educativo os Comitês têm como principais objetivos estabelecer uma rede de vigilância dos óbitos infantis ocorridos nos municípios a partir da estratégia de "evento sentinela", avaliar e corrigir, Quando necessário, a causa básica desses óbitos bem como identificar os fatores determinantes e propor intervenções através de medidas educativas e informativas visando a prevenção, promoção e reorganização da assistência à saúde.

No Paraná 1999 foi o primeiro ano de efetiva atuação dos Comitês Regionais de Prevenção da Mortalidade Infantil ${ }^{(10)}$. Estão organizados no estado 22 Comitês Regionais e diversos comitês municipais, alguns atuando de forma articulada com os Comitês de Prevenção da Mortalidade Materna.

CRPMI da $15^{\mathrm{a}}$ RS é formado por representantes de diversos setores da sociedade: da SMS, sociedades científicas, conselhos profissionais e de instituições de ensino como a Universidade Estadual de Maringá. O CRPMI inicia o processo de investigação a partir da declaração de óbito (DO) Que, até o ano de 2004, eram triadas selecionando-se os óbitos de recém-nascidos com peso ao nascer igual ou superior a $1000 \mathrm{~g}$. Os municípios da $15^{\mathrm{a}} \mathrm{RS}$ encaminham à $15^{\mathrm{a}} \mathrm{RS}$ os documentos para análise do óbito infantil de seus residentes, além do relatório da visita domiciliar realizada junto à família, dados sobre o pré-natal, parto, a história de vida e as circunstâncias do óbito da criança. O CRPMI analisa os documentos disponíveis, estabelecendo um itinerário desde o prénatal até o nascimento da criança reconstituindo a historia, analisando as complicações Que levaram ao óbito determinando a redutibilidade, os critérios de redutibilidade, a responsabilidade do óbito, as medidas de prevenção, além de estabelecer a causa básica Que pode ou não coincidir com a registrada na declaração de óbito. A análise detalhada possibilita melhorar o conhecimento das circunstâncias de ocorrência do óbito infantil e identificar os fatores de risco.

As informações coletadas e as correções realizadas pelo CRPMI são digitadas e inseridas em um sistema de informações próprio do estado do Paraná denominado Sistema de Informação de Mortalidade Infantil (SIMI). Após digitação em cada Regional de Saúde os dados são encaminhados à Secretaria de Saúde do Estado do Paraná.

O SIMI é um sistema de armazenamento de dados desenvolvido pela Secretaria Estadual de Saúde do Paraná (SESA) em 2000 para digitação das principais conclusões das análises dos óbitos infantis pelos Comitês Regionais, o Qual permite agilizar a obtenção dos dados via rede intranet da Secretaria de Estado da Saúde ${ }^{(10)}$. O SIMI foi criado com objetivo de auxiliar na tabulação de dados das investigações dos óbitos infantis feitas nas Regionais de Saúde, facilitando a análise e subsidiando as ações localizadas nos municípios, nas Regionais e ações desencadeadas pelos Comitês Regionais e Comitê Estadual|(1).

Existem também na $15^{\mathrm{a}} \mathrm{RS}$ os Comitês setoriais de prevenção à mortalidade infantil no município sede Que é Maringá e em dois hospitais com leitos de UTI neonatal. Essas instituições de saúde realizam pré-analise e pré-investigação de óbitos infantis ocorridos internamente ficando em seu poder os prontuários de internação da mãe e do bebê. São enviadas à $15^{\mathrm{a}} \mathrm{RS}$ as pré-análises, as ficha de investigação e de visita domiciliar para posterior verificação, conclusão e consolidação do caso em reunião mensal do Comitê Regional, para em seguida proceder a digitação no SIMI e respectivo encaminhamento ao Comitê Estadual. As investigações e análise do Comitê permitem a correção da causa básica do óbito infantil no Sistema de Informação sobre Mortalidade (SIM/MS) o que certamente melhora a qualidade dos dados no Sistema oficial de mortalidade.

\section{O RELATO DE EXPERIÊNCIA}

A apresentação dos acadêmicos o reconhecimento do local e atividades desenvolvidas na Sessão de Epidemiologia da $15^{\mathrm{a}} \mathrm{RS}$ é fase inicial Quando são incorporados novos integrantes no projeto. Em seguida ocorre a familiarização com os documentos como a declaração de óbito (DO), a Declaração de Nascido Vivo (DN), o cartão da gestante, a ficha de investigação do óbito infantil e prontuários de atendimento ambulatorial e hospitalar da mãe e da criança. Todos os documentos necessários são reunidos para a realização posterior das investigações. Essa fase de montagem do processo com os documentos e formulários da historia do atendimento do pré-natal e do parto e do bebê é lenta, pois os municípios e instituições levam algum tempo até atenderem às solicitações do Comitê Regional.

Outra dificuldade do Comitê tem sido a de compreender a letra e as informações nos documentos Que nem sempre são satisfatórios ou colocados de forma clara nos registros e formulários incluídos no processo. A falta de documentos ou de informações claras gera dificuldades no momento das análises. Muitas vezes surgem dúvidas como, por exemplo, a ordem temporal dos fatos ocorridos relativos ao óbito em investigação. Por isso durante a análise são acionadas as instituições envolvidas para Que estas enviem mais informações e documentos da mãe e do bebê. Nem sempre é possível acrescentar informações e documentos no processo, ou pela demora no atendimento às demandas do Comitê ou pelo simples fato desses documentos não existirem nos hospitais ou nas Unidades Básicas de Saúde. Em relação às entrevistas domiciliares, Que muitas vezes acrescentam informações imprescindíveis para a investigação ocorre, em alguns casos impossibilidade de sua realização, ou por motivo de mudança ou inexistência de endereço ou ainda por recusa por parte dos familiares da criança em responder as perguntas do profissional de saúde.

Vale comentar aspectos na operação do SIMI em rede intranet, já que a informatização é fator imprescindível para o adeQuado andamento das digitações, análises e encaminhamento dos dados. Muitas vezes os eQuipamentos de informática não estão atualizados 
ou estão em manutenção ou atualização o Que compete para uma alimentação do SIMI de forma ágil e com Qualidade.

Algumas mudanças e progressos foram observados com a participação articulada do Departamento de Enfermagem nas atividades no Comitê. Após o início das atividades do projeto de extensão tem sido rotina nas reuniões do Comitê Regional a presença de profissionais de saúde de outros municípios, principalmente da equipe de enfermagem das Unidades Básicas de Saúde onde a gestante fez pré-natal. Essa participação é parte do rol de atribuições dos Comitês, de informar aos serviços os resultados das análises, além de atender a necessidade de descentralizar essas discussões aos serviços de saúde Que atendem a gestante e a criança. O envolvimento da equipe de enfermagem nos estudos de caso de cada óbito infantil principalmente os ocorridos no seu município contribui para a sensibilização e aprimoramento do profissional no atendimento a mulher e a criança.

Outra atividade estimulada na rotina de trabalho e na atribuição do CRPMI são as visitas periódicas Que tem sido feitas aos serviços de epidemiologia e as equipes de saúde, em especial de enfermagem, nos municípios pertencentes à $15^{\mathrm{a}} \mathrm{RS}$. Tais visitas têm como objetivo principal sensibilizar esses profissionais da necessidade da Qualidade da assistência ao pré-natal, encaminhamento e referência para gestantes e bebê de risco além de assessoria, esclarecimento de dúvidas Quanto á utilização dos formulários e busca de informações adicionais relevantes para as investigações em curso.

Como as análises e investigações dos óbitos infantis não estavam bem sistematizadas pelo Comitê para garantir sua Qualidade foi elaborado um roteiro com todas as informações Que devem ser citadas em ordem temporal dos fatos Que compõem a história do óbito. Esse roteiro é distribuído aos Comitês setoriais hospitalares e também aos municípios durante as visitas, tanto para divulgar o trabalho do Comitê Regional como também para estimular cada município elaborar suas análises montando um grupo de trabalho e oficializando os Comitês municipais.

A ficha de investigação do óbito infantil também foi adaptada, pois a principio eram duas fichas diferentes, uma para análise dos óbitos ocorridos no período neonatal e outra para os ocorridos no período pós-neonatal. A utilização de duas fichas diferentes começou a ser Questionada pelo Comitê, pois gerava dúvidas no seu preenchimento. Assim as fichas foram unificadas e foram acrescentados alguns dados indispensáveis como o financiamento do pré-natal e parto, se em caso de risco gestacional houve encaminhamento, além de modificações na forma de apresentação objetivando facilitar a coleta de dados pelo entrevistador. $\mathrm{Na}$ reformulação da ficha de investigação foi acrescentado mais espaço para os dados da entrevista domiciliar, já Que essas informações Quando coletadas podem elucidar circunstâncias e fatos não constantes nos prontuários das UBS e dos hospitais. Considera-se Que as impressões do entrevistado, na maior parte das vezes da mãe ou de algum familiar, sobre o óbito infantil traz uma compreensão necessária para os serviços de saúde Que extrapola o significado numérico e documental da historia do óbito, consistindo fundamental subsidio para o adequado planejamento de políticas e ações em saúde ${ }^{(12)}$.

\section{Análise dos Dados da Ficha de Investigação do Óbito Infantil} em 2005

Um desafio para intensificar e avançar o trabalho do Comitê e do projeto de extensão aQui relatado e Que já vem sendo enfrentado consiste em aprimorar a integração Universidade/serviços de saúde no sentido de envolver e sensibilizar a equipe com o trabalho de investigação e devolver, de forma sistemática os resultados das investigações. Para isso é necessário melhorar a Qualidade das análises e dos relatórios finais e conseqüentemente dos SIS Que são alimentados com os dados dessas análises.

Com o objetivo conhecer a Qualidade das informações e dados registrados nos documentos foi feito um levantamento dos dados constantes na ficha de investigação do óbito infantil do ano de 2005 apresentados nas Tabelas 1, 2, e 3.

A ficha de investigação é um formulário Que reúne dados de identificação da criança, do óbito, do pré-natal, do cartão da gestante, do parto, dados ambulatoriais e hospitalares da gestante e da criança e principalmente os dados da entrevista domiciliar. Essa ficha deve ser preenchida no município de residência da criança por algum profissional de saúde em geral das UBS, da Equipe de saúde da Família ou do setor de Epidemiologia das Secretarias Municipais.

Observou-se que, dos dados declarados $56,31 \%$ dos partos foram financiados pelo SUS, 51 ,46\% das mães realizaram o pré-natal em Unidade Básica de Saúde e 55,34\% das famílias recebiam até 3 salários mínimos mensais (Tabela 1). Entretanto chama atenção o alto percentual de informações não declaradas. O financiamento do parto não foi declarado em 33\% das vezes, o local do pré-natal em $17,5 \%$, a renda familiar em $35 \%$ e informações se a mãe tem companheiro ou não ficaram sem ser declaradas em 18,5\% das vezes.

lá para os dados do recém-nascido foi observado melhor preenchimento na ficha de investigação, provavelmente pela maior ocorrência de óbitos no período neonatal precoce sem o bebe ter tido alta hospitalar. Nesses casos o prontuário de internação do bebê seria a fonte para preenchimento da ficha de investigação além da existência dos Comitês nos dois hospitais com UTI neonatal em Maringá. Como Maringá é a sede da $15^{\text {a }}$ RS e conta com os serviços de saúde especializados, é referencia para os 30 municípios da RS para as gestações e bebês de risco. Apenas a informação se o recém-nascido foi considerado de risco observou-se $75,7 \%$ de não declaração (Tabela 2) provavelmente pelo mesmo motivo, em razão do óbito ter ocorrido no período neonatal ainda em ambiente hospitalar.

A Tabela 3 destaca o Quanto as informações sobre a história da gestação estão ausentes das fichas de investigação do óbito infantil, provavelmente devido à falta dos documentos nos prontuários de análises. É freqüente e bem conhecida a falta das informações nos prontuários da gestante e puérpera sobre as intercorrências na gestação.

Entre os documentos importantes e indispensáveis para compor o prontuário de análise do óbito infantil merece destaQue o cartão da gestante. Ainda não é dada a devida importância a esse documento pela equipe de saúde, pois muitas vezes os dados não são registrados no cartão e também a gestante chega ao hospital no momento do parto sem o documento.

Para avaliar a adeQuação do processo da assistência pré-natal pelo Sistema Único de Saúde em Juiz de Fora-MG foi feita uma auditoria em 2002 e foi encontrado Que 7,9\% das parturientes não apresentaram seus cartões de gestante durante a internação ${ }^{(13)}$. Em Salvador para o ano de 2001 estudo revelou Que 29,3\% das gestantes Que procuraram atendimento em uma maternidade não 
Tabela 1. Informações da ficha de investigação do óbito infantil, segundo financiamento do pré-natal, parto, renda familiar e situação conjugal da mãe. CRPMI, 15ª Regional de Saúde de Maringá-PR, 2005.

\begin{tabular}{lcc}
\hline Variáveis & $\mathrm{n}$ & $\%$ \\
\hline Financiamento parto & 58 & 56,31 \\
SUS & 8 & 7,77 \\
Particular & 3 & 2,91 \\
Parto domiciliar & 34 & 33,01 \\
Sem informação & & \\
Local do Pré-natal & 53 & 51,46 \\
UBS & 21 & 20,39 \\
Particular & 10 & 9,71 \\
Não fez Pré-natal & 1 & 0,97 \\
UBS +Particular & 18 & 17,48 \\
Sem informação & & \\
Renda familiar mensal & 31 & 30,10 \\
até 1 & 26 & 25,24 \\
2 a 3 & 8 & 7,77 \\
4 a 5 & 1 & 0,97 \\
6 ou mais & 37 & 35,92 \\
Sem informação & & \\
Companheiro & 72 & 69,90 \\
Sim & 21 & 20,39 \\
Não & 19 & 18,45 \\
Sem informação & &
\end{tabular}

Tabela 2. Informações da ficha de investigação do óbito infantil sobre o Recém Nascido. Comitê de Prevenção da Mortalidade Infantil, 15a Regional de Saúde de Maringá-PR, 2005.

\begin{tabular}{lcc}
\hline Variáveis & $\mathrm{n}$ & $\%$ \\
\hline Internação do recém-nascido & 65 & \\
Sim & 36 & 63,11 \\
Não & 2 & 34,95 \\
Sem informação & & 1,94 \\
Internação em UTI & 60 & \\
Sim & 39 & 58,25 \\
Não & 4 & 37,86 \\
Sem informação & & 3,88 \\
Considerado recém-nascido de risco & 5 & \\
Sim & 20 & 4,85 \\
Não & 78 & 19,42 \\
Sem informação & & 75,73 \\
\hline
\end{tabular}

apresentaram o cartão de gestante ${ }^{(14)}$. Tais resultados mostram, em diferentes proporções, Que ainda existem dificuldades e falta de informação na atribuição da importância dada ao Cartão da gestante. A rotina de preenchimento dos cartões é dificultada pela multiplicidade de modelos utilizados. Foi verificado Que faltam de registros de informações do pré-natal como vacinação antitetânica, curvas do crescimento uterino, idade gestacional, data da última menstruação, entre outros ${ }^{(13)}$.

Essa breve análise da Qualidade dos dados da ficha de investigação remete à importância do caráter educativo dos Comitês na orientação e constante diálogo com os municípios e equipes de saúde que fazem a entrevista domiciliar a procurarem ao entrevistar a família, responder todas essas informações e acrescentar outras Que não constam nos prontuários. 
Tabela 3. Informações da ficha de investigação do óbito infantil, sobre variáveis da gestação. Comitê de Prevenção da Mortalidade Infantil, 15ª Regional de Saúde de Maringá-PR, 2005.

\begin{tabular}{|c|c|c|}
\hline Variáve is & $\mathrm{N}^{\circ}$. & $\%$ \\
\hline \multicolumn{3}{|l|}{ Ia consulta pré-natal } \\
\hline $1^{\circ}$ Trimestre & 30 & 29,13 \\
\hline $2^{\circ}$ Trimestre & 19 & 18,45 \\
\hline $3^{\circ}$ Trimestre & 2 & 1,94 \\
\hline Não fez pré-natal & 10 & 9,71 \\
\hline Sem informação & 42 & 40,78 \\
\hline \multicolumn{3}{|l|}{ Risco gestacional } \\
\hline Sim & 20 & 19,42 \\
\hline Não considerada de risco & 43 & 41,75 \\
\hline Sem informação & 40 & 38,83 \\
\hline \multicolumn{3}{|l|}{ Motivo do risco* } \\
\hline Hipertensão & 6 & 5,83 \\
\hline Mãe adolescente & 5 & 4,85 \\
\hline Problemas com o feto & 3 & 2,91 \\
\hline Diabetes & 2 & 1,94 \\
\hline Outros & 8 & 7,77 \\
\hline Não considerada de risco & 43 & 41,75 \\
\hline Sem informação & 40 & 38,83 \\
\hline \multicolumn{3}{|l|}{ Complicações na gestação } \\
\hline Sim & 59 & 57,28 \\
\hline Não & 18 & 17,48 \\
\hline Sem informação & 26 & 25,24 \\
\hline \multicolumn{3}{|l|}{ Tipos de complicação* } \\
\hline TPP & 24 & 24,00 \\
\hline Infecção urinária & 14 & 14,00 \\
\hline Ameaça de aborto & 13 & 13,00 \\
\hline Hipertensão & 13 & 13,00 \\
\hline Hemorragia & 11 & 11,00 \\
\hline Diabetes & 2 & 2,00 \\
\hline Outras & 23 & 23,00 \\
\hline
\end{tabular}

\section{CONSIDERAÇÕES FINAIS}

O trabalho no Comitê e o contato com os sistemas de informação vêm proporcionando à equipe participante do projeto de extensão e da $15^{\mathrm{a}} \mathrm{RS}$ a compreensão do funcionamento, da lógica e importância das informações em saúde e de cada banco de dados eue compõem os Sistemas de Informação do SUS.

Outro avanço do projeto consiste no conhecimento dos participantes de todo o arsenal de formulários, guias de encaminhamento, receituários, o próprio prontuário do paciente, fichas de notificação e fichas de investigação. Além de conhecer os SIS está sendo possível compreender a complexidade do processo de trabalho nos serviços de saúde desde a geração do dado, digitação, compilação, Qualidade e potencialidade de utilização. Nesse sentido, a necessidade de capacitação da eQuipe de saúde, em especial do enfermeiro deve ser constante, primeiro para uma melhor assistência a população e para um registro dos dados com
Qualidade e ainda, para compreender o potencial de utilização dessas informações para planejar e priorizar as atividades na comunidade.

Aspecto fundamental para a formação de recursos humanos em saúde é a compreensão dos eventos Que geram os dados e em seguida a análise gerando a informação. O contato dos alunos de enfermagem com a história do óbito infantil remete à compreensão da existência da gestante na comunidade inscrita em programa de atendimento ao pré-natal, ao nascimento de uma criança, a ocorrência de determinada doença, de uma internação hospitalar ou, em última instância, a ocorrência do óbito Que são objetos de trabalho nos serviços de saúde. Esse envolvimento, também relatado em outro estudo ${ }^{(15)}$ amplia a compreensão do aluno sobre o Quadro sanitário da comunidade, das desigualdades intraurbanas, dos aspectos relacionados à assistência, da pratica profissional e do acesso aos serviços, incentivando sua formação mais comprometida com a saúde coletiva.

A investigação dos óbitos infantis pelo Comitê da $15^{\text {a }}$ RS estabelecendo as causas desses óbitos e considerando reduzíveis 
mais de 70\% deles, aponta para o grande desafio aos serviços e gestores na formulação de políticas publicas voltadas para a atenção à mulher e à criança.

O reconhecimento da importância da presença de alunos de graduação em instituições públicas de saúde vem aumentando dando maior visibilidade aos projetos de extensão universitária. Atividades Que integram a educação e serviços de saúde devem ser valorizadas, pois propiciam formação mais adeQuada, integrando um conjunto de estratégias que buscam a capacitação de trabalhadores para o SUS ${ }^{(16)}$. Essa integração viabiliza ainda estratégias para o ensino docente, desenvolve no aluno potencialidades para enfatizar a compreensão dos conteúdos no processo de aprendizagem e fortalece a prática os saberes pedagógicos ${ }^{(17)}$.

Assim os projetos de extensão devem ser estimulados e valorizados, pois estabelecem parcerias e intercâmbios de profissionais das universidades e dos serviços em ambiente propício para Que os problemas da prática cotidiana possam ser incorporados às atividades de pesquisa. Da mesma forma possibilitam Que os resultados de pesquisas e investigações acadêmicas sejam utilizados para aprimoramento das políticas e práticas dos serviços de saúde.

\section{REFERÊNCIAS}

I. Peterlini OLG, Zagonel IPS. O sistema de informação utilizado pelo enfermeiro no gerenciamento do processo de cuidar. Texto Contexto Enferm 2006; 15(3): 418-26.

2. Ministério da Saúde (BR). IV Plano Diretor para o Desenvolvimento da Epidemiologia no Brasil: Epidemiologia nas Políticas, Programas e Serviços de Saúde. Rev Bras Epidemiol 2005; 8(Supl. 1): 28-39.

3. Mathias TAF, Mello Jorge MHP. Sistema de informações sobre mortalidade: análise da Qualidade dos dados para o município de Maringá, estado do Paraná, Brasil. Acta Scientiarum 200 I; 23(3): 759-65.

4. Moraes IHS. Informações em Saúde: da prática fragmentada ao exercício da cidadania. São Paulo: Hucitec/Abrasco; 1994.

5. Drumond I. Epidemiologia nos Municípios: muito além das normas. São Paulo: Hucitec; 2003.

6. Ministério da Saúde (BR). Fundação Nacional de Saúde. Guia de Vigilância Epidemiológica/FNS. 5a ed. Brasília: FUNASA, 2002.

7. Ministério da Saúde (BR). Departamento de Atenção Básica. Guia prático do Programa de Saúde da Família-Parte I. Brasília: Ministério da Saúde; 200I.

8. Vidal AS, Frias PG, Barreto FMP, Vanderlei LCM, Felisberto E. Óbitos infantis evitáveis em hospital de referência estadual do Nordeste brasileiro. Rev Bras Saúde Matern Infant 2003; 3(3): 28I-9.

9. Ministério da Saúde (BR). Manual dos Comitês de Prevenção do Óbito Infantil e Fetal. Brasília: Ministério da Saúde; 2004.

10. Mansano NH, Mazza VZ, Soares VMN, Araldi MAR, Cabral
VLM. Comitês de prevenção da mortalidade infantil no Paraná, Brasil: implantação e operacionalização. Cad Saúde Pública 2004; 20 :329-32.

I I. Vianna RCXF, Trindade CM, Moro CMC. Sistema de Investigação da Mortalidade Infantil - SIMI. [citado em: 24 fev 2008]. Disponível em: URL: http://www.saude.pr.gov.br/ftp/CEPMMI/ SIMI.pdf

12. Goulart LMHF, Somarriba MG, Xavier CC. A perspectiva das mães sobre o óbito infantil: uma investigação além dos números. Cad Saúde Publica 2005; 2 I(3): 715-23.

13. Coutinho T, Teixeira MTB, Dain S, Sayad ID, Coutinho LM. Adequação do processo de assistência pré-natal entre as usuárias do Sistema Único de Saúde em Juiz de Fora-MG. Rev Bras Ginecol Obst 2003; 25(10): 717-724.

14. Lima BGC, Pimentel CO. Qualidade do pré-natal de gestantes assistidas numa maternidade pública de Salvador, 2000-200 I Rev Baiana Saúde Pública 2003; 27(1/2): 50-59.

15. Malta DC, Lansky S, França E, Xavier CC, Silva AMDA, Rezende EM. Prevenção do óbito infantil e perinatal em Belo Horizonte e a avaliação das investigações realizadas. In: Anais do $6^{\circ}$. Encontro de Extensão UFMG. 2003; Belo Horizonte (MG), Brasil. Belo Horizonte: UFMG; 2003. p. 228-34.

16. Almeida LPG, Ferraz CA. Políticas de formação de recursos humanos em saúde e enfermagem. Rev Bras Enferm 2008; 6I(1): 31-5.

17. Valsecchi EASS, Nogueira MS. Estratégias de ensino utilizadas na disciplina de fundamentos de enfermagem. Ciência Cuidado Saúde 2006; 5 (Supl. I): I13-8. 